\title{
Ansiedade e insônia: relato de caso sobre a importância do foco de tratamento na
}

\section{qualidade do sono}

\author{
Anxiety and insomnia: case report on the importance of treatment focus on sleep quality \\ Ansiedad e insomnio: reporte de un caso sobre la importancia del enfoque del tratamiento en la \\ calidad del sueño
}

Recebido: 17/11/2021 | Revisado: 29/11/2021 | Aceito: 30/11/2021 | Publicado: 04/12/2021

Alan das Neves Junior
ORCID: https://orcid.org/0000-0001-9187-9484
Universidade para o Desenvolvimento da Região do Pantanal, Brasil
E-mail: alandasnevesjr.ajdn@ gmail.com
José Carlos Rosa Pires de Souza
ORCID: https://orcid.org/0000-0003-4460-3770
Universidade Estadual de Mato Grosso do Sul, Brasil
E-mail: josecarlossouza@ uol.com
Clayton Peixoto
ORCID: https://orcid.org/0000-0002-5334-3467
Universidade Federal do Rio de Janeiro, Brasil
E-mail: claytonpeixoto@yahoo.com.br

\section{Resumo}

Introdução: os transtornos de ansiedade podem estar relacionados aos distúrbios do sono como fatores etiológicos ou complicações; o objetivo deste relato foi discutir o caso de uma paciente com Transtorno de Ansiedade Generalizada e insônia, que teve melhora dos seus sintomas ansiosos com o tratamento da insônia. O foco de seu tratamento foi a qualidade do sono. Metodologia: Trata-se de uma mulher de 32 anos com maus hábitos de Higiene do Sono, que foi anteriormente tratada e que, embora tenha melhorado seus sintomas ansiosos, não conseguiu obter melhora na qualidade do sono. Após uma intervenção com Terapia Cognitivo-comportamental, voltada para o tratamento da insônia, houve melhora tanto da qualidade de sono quanto dos sintomas ansiosos. Conclusão: Este caso aponta para a importância do cuidado com os hábitos de sono dos pacientes, mesmo aqueles cuja queixa primária seja outro transtorno mental.

Palavras-chave: Transtorno de ansiedade generalizada; Distúrbio do sono; Insônia; Higiene do sono.

\begin{abstract}
Introduction: anxiety disorders can be related to sleep disorders as etiological factors or complications; the purpose of this report was to discuss the case of a patient with Generalized Anxiety Disorder and insomnia, who had an improvement in her anxious symptoms with the treatment of insomnia. Her treatment focus was on quality of sleep.

Methodology: This is a 32-year-old woman with poor sleep hygiene habits, who was previously treated and who, although her anxiety symptoms improved, was unable to improve her sleep quality. After an intervention with Cognitive-behavioral Therapy, aimed at the treatment of insomnia, there was an improvement in both sleep quality and anxiety symptoms. Conclusion: This case points to the importance of caring for patients' sleep habits, even those whose primary complaint is another mental disorder.
\end{abstract}

Keywords: Generalized anxiety disorder; Sleep disorder; Insomnia; Sleep hygiene.

\section{Resumen}

Introducción: los trastornos de ansiedad pueden relacionarse con los trastornos del sueño como factores etiológicos o complicaciones; El objetivo de este informe fue discutir el caso de una paciente con trastorno de ansiedad generalizada e insomnio, que mejoró sus síntomas de ansiedad con el tratamiento del insomnio. El foco de su tratamiento fue la calidad del sueño. Metodología: Se trata de una mujer de 32 años con malos hábitos de higiene del sueño, que fue previamente tratada y que a pesar de haber mejorado sus síntomas de ansiedad, no pudo mejorar su calidad de sueño. Tras una intervención con Terapia Cognitivo-Conductual, dirigida a tratar el insomnio, se observó una mejora tanto en la calidad del sueño como en los síntomas de ansiedad. Conclusión: Este caso apunta a la importancia de cuidar los hábitos de sueño de los pacientes, incluso aquellos cuya principal queja es otro trastorno mental.

Palabras clave: Trastorno de ansiedad generalizada; Desorden del sueño; Insomnio; Higiene del sueño. 


\section{Introdução}

As queixas relacionadas ao sono são muito comuns na prática clínica e merecem atenção devido ao seu impacto na vida das pessoas afetadas. Müller e Guimarães (2007) apontam para o prejuízo funcional que os transtornos do sono podem causar, entre eles, riscos de acidentes de trânsito, absenteísmo e presenteísmo no trabalho, agravamento de problemas de saúde, aumento do risco de distúrbios mentais e comprometimento na qualidade de vida Entre os transtornos do sono mais comuns está a insônia, que segundo dados epidemiológicos de diferentes estudos, afeta entre 10 e $40 \%$ da população geral (Prado, 2016; Thorpy, 2012). As queixas de insônia incluem dificuldades de iniciar e/ou manter o sono, contendo geralmente prolongados períodos de vigília noturna e/ou quantidades insuficientes de sono durante a noite (Pafume et. al., 2014; American Psychiatry Association, 2013, p.189).

Os transtornos de ansiedade estão entre os quadros psiquiátricos mais prevalentes, estimando-se que 9 a $15 \%$ da população apresentem este quadro ao longo da vida (American Psychiatry Association, 2013, p.225). Os transtornos de ansiedade se caracterizam pelo medo e ansiedade excessivos relacionados a objetos ou situações (Barros et. al., 2020), contudo existem situações em que o indivíduo não consegue identificar de forma clara objetos/situações ansiogênicas, prevalecendo um sentimento difuso de ansiedade. Entre os problemas mais comuns causados pelos transtornos de ansiedade, bem como outros transtornos mentais estão os problemas relacionados ao sono e vice-versa (American Psychiatry Association, 2013, p.368; Barros et. al., 2020).

Apesar da inter-relação entre os transtornos mentais e os transtornos do sono e apesar do paciente nem sempre conseguir relatar com precisão qual é o seu principal problema, aspectos relacionados à higiene do sono, que são de grande importância diagnóstica, nem sempre são adequadamente avaliados. Consequentemente, a introdução da higiene do sono deixa de ter prioridade no tratamento de indivíduos com transtornos mentais que se queixam de dificuldades relacionadas ao sono. Este trabalho teve por objetivo apresentar o caso de uma mulher com diagnóstico de transtorno de ansiedade generalizada comórbido com transtorno de insônia; e que teve melhora dos seus sintomas ansiosos com a melhoria da qualidade do sono.

\section{Metodologia}

O presente estudo, de natureza qualitativa e descritiva, trata-se de um relato de caso decorrente de prática profissional e as informações aqui contidas foram obtidas durante o acompanhamento psicológico da paciente relatada, bem como através de revisão de literatura e discussão para consenso entre os autores especialistas. Este trabalho foi realizado respeitando toda a normatização de ética em pesquisa vigente, com base na Declaração de Helsinque (1964), e na Resolução 196 do Conselho Nacional de Saúde (CNS/196) que estabelece as normas para a realização de relatos de caso. Foi oferecido a paciente relatada um termo de consentimento livre e esclarecido, o qual a mesma leu e assinou consentindo a divulgação do caso desde que resguardado o seu direito ao anonimato.

\section{Resultados}

\subsection{Relato de caso}

Uma mulher, 32 anos, advogada, casada, sem filhos, procurou atendimento psicológico por orientação de seu psiquiatra, a fim de se tratar dos seus sintomas ansiosos decorrentes do transtorno de ansiedade generalizada (CID-10: F41.1). Relatou sintomas de ansiedade, sonolência diurna excessiva, dificuldades de iniciar o sono, sono polifásico e não reparador, cansaço mesmo depois de uma noite de sono, dores de cabeça e irritabilidade. Segundo relatou, este quadro estava prejudicando seriamente a sua relação conjugal. Os seus sintomas de ansiedade começaram a lhe incomodar há, aproximadamente, sete anos atrás, logo após o casamento e se intensificaram há quatro anos. Associa o aumento da ansiedade 
à pressão familiar para passar em um concurso público e, consequentemente, ter a estabilidade profissional necessária para gerar um filho. Negou crises de pânico, ideias obsessivas e intrusivas, comportamento estereotipados e compulsivos; não apresentou sintomas depressivos proeminentes ou sintomas de outros transtornos mentais. Questionada sobre apneia do sono, relatou não apresentar esse problema, informação confirmada pelo cônjuge.

A paciente faz exames de rotina anualmente; o último foi realizado, aproximadamente, há três meses antes da primeira consulta psicológica. Não relatou qualquer alteração que pudesse justificar os sintomas atuais, como por exemplo, hipertensão arterial ou obesidade. Pratica atividades físicas de 3 a 5 vezes por semana, mantém alimentação saudável, não fuma e nem consome bebidas alcoólicas, entretanto fazia uso regular de café e energéticos para se manter acordada ao longo do dia, especialmente quando sentia muito sono. Segundo relatou, esses períodos de sonolência diurna excessiva ocorriam no trabalho, principalmente após as 15 horas.

Sua rotina diária e semanal consistia em acordar às seis horas da manhã, tomar café às 06h30min e entrar no trabalho às 07h30min. Seu horário de almoço era de onze às treze. Relatou almoçar no serviço e dormir em um sofá de sua sala por aproximadamente setenta minutos antes de voltar para o turno vespertino. Chegava do trabalho às $18 \mathrm{~h} 30 \mathrm{~min}$ e por volta das dezenove começa a estudar. Por volta das 20h30min, quando já se sentia muito cansada para continuar estudando, fazia um lanche e ia para academia de musculação entre vinte e uma e vinte e duas horas. Jantava ao voltar da academia, por volta das $22 \mathrm{~h} 15 \mathrm{~min}$ e ia para cama às 23 horas. Entretanto quase nunca conseguia dormir de imediato. Por conta disso, ficava assistindo TV ou vendo o celular até dar sono, o que normalmente ocorria por volta de uma ou duas horas da manhã.

Segundo relata, o seu psiquiatra disse que ela sofria de ansiedade em um nível moderado e que precisava fazer psicoterapia, contudo ele lhe passaria uma medicação (Hemitartarato de Zolpidem 10mg), que teria a função de auxiliá-la em relação ao sono. Contudo, a paciente se mostrou resistente ao uso de medicação, pois relatou medo de ficar dependente da mesma.

Há aproximadamente três anos, antes de procurar atendimento psiquiátrico, relata ter feito tratamento psicológico na abordagem cognitivo-comportamental com vistas a tratar sintomas de ansiedade. Depois de três meses de acompanhamento psicológico, relatou que seus sintomas de ansiedade remitiram de forma bastante satisfatória, contudo o seu sono apresentou apenas uma pequena melhora.

\subsection{Instrumentos de avaliação, diagnóstico e tratamento}

Para a complementação da anamnese, a paciente preencheu o seu Diário do Sono com questões simples relacionadas aos horários de se deitar e despertar, quantidade e causas de despertares noturnos, entre outros aspectos; como também, foi feita a aplicação da Escala de Beck de Ansiedade (BAI), a Escala de Sonolência Epworth (ESE) e o Índice de Gravidade de Insônia (IGI). O BAI é um instrumento autoaplicável, de múltiplas escolhas, com 21 questões que podem ser pontuadas de 0 a 3. Pontuações de 0 a 10 neste instrumento indicam ansiedade mínima, 11 a 19 ansiedade leve, 20 a 30 ansiedade moderada e 31 ou mais ansiedade severa (Leyfer, Ruberg \& Woodruff-Borden, 2006).

Segundo Amorim (2017), a ESE avalia a probabilidade de o indivíduo cochilar em situações rotineiras como ler e assistir televisão. A pontuação varia de zero a três pontos em cada situação analisada: o zero representa não cochilar nunca; o um significa pequena chance de cochilar; o dois indica moderada chance de cochilar; e o três sugere grande chance de cochilar".

O mesmo autor refere que o IGI "é um questionário de sete itens que avalia a gravidade e o impacto da insônia no mês anterior à sua aplicação; o instrumento contém sete perguntas com pontuação que varia de zero a quatro. Os itens específicos avaliam dificuldades como início do sono, manutenção do sono, despertar matinal, entre outros. A pontuação total mais elevada indica distúrbio grave no sono. 
A paciente apresentou 21 pontos no BAI, 12 na ESE e 13 na IGI. Considerando o diagnóstico médico, a avaliação psicológica e os resultados das escalas aplicadas, ficou identificado que a paciente apresentava um quadro moderado de ansiedade e sonolência diurna excessiva, decorrente da insônia. Estes resultados implicaram na reflexão sobre se a ansiedade estaria causando a insônia ou se a insônia estaria causando a ansiedade, uma vez que os hábitos de vida poderiam estar interferindo, seriamente, no sono da paciente e, consequentemente, exacerbando os sintomas ansiosos. Por conta disso, e também por conta do histórico prévio de tratamento de sintomas de ansiedade, optou-se por uma terapêutica que contemplasse ambos os transtornos apresentados, mas com um foco inicial direcionado para a resolução dos problemas de insônia. Ficou acordado com a paciente 12 sessões de terapia cognitivo-comportamental (TCC), uma vez por semana, com 50 minutos de duração.

\subsection{Fundamentação teórica da opção terapêutica adotada}

A TCC é uma abordagem terapêutica estruturada e de curta duração, direcionada para a resolução de problemas atuais e a modificação de pensamentos e comportamentos disfuncionais 10. Segundo Beck 2016 "o modelo cognitivo propõe que o pensamento disfuncional (que influencia o humor e o pensamento) é comum a todos os transtornos psicológicos. Quando as pessoas aprendem a avaliar seu pensamento de forma mais realística e adaptativa, elas obtêm uma melhora em seu estado emocional e no comportamento".

De forma geral, o processo terapêutico baseado na TCC tem como um dos objetivos a psicoeducação, visando com que o paciente consiga perceber os mecanismos de funcionamento de sua patologia; e assim podendo engajá-lo ativamente no processo terapêutico (Beck 2016; Manfro et. al. 2008; Matta et. al 2010).

O objetivo da TCC nos transtornos de ansiedade consiste em readequar a cognição e, por consequência, as emoções e o comportamento de pessoas acometidas por esse distúrbio. Uma pessoa ansiosa costuma apresentar uma tríade cognitiva de pensamentos disfuncionais que incluem uma visão de um "eu" vulnerável, um mundo perigoso e um futuro incerto (Manfro et. al. 2008; Matta et. al 2010).

No tratamento da insônia, a TCC tem por objetivos identificar e confrontar os pensamentos disfuncionais específicos relacionados a insônia (Margis 2015). Segundo Harvey (2002), o modelo cognitivo da insônia envolve um conjunto de crenças e comportamentos disfuncionais relacionados ao sono; na hora de dormir os indivíduos com insônia apresentam uma atividade cognitiva excessiva e negativa relacionada ao sono. Este comportamento gera excitação e angústia, que por sua vez produz aumento na atenção seletiva e no monitoramento de qualquer sinal; este que indica problemas relacionados ao sono, que por sua vez aumenta a excitação e a angústia e produz uma distorção na percepção da deficiência em dormir. Este último também contribui para aumentar ainda mais a excitação e a angústia e, consequentemente, provocar um déficit real de sono. A TCC "engloba diferentes estratégias, tal como terapia de controle de estímulos, restrição de sono, higiene do sono, treinamento em relaxamento e terapia cognitiva". Ou seja, o terapeuta no processo de tratamento da insônia deve se preocupar em introduzir mudanças de hábitos de vida, que estejam influenciando na manutenção do transtorno do sono; além de readequar o pensamento do paciente (Margis 2015).

\subsection{Tratamento e evolução}

Na primeira sessão de TCC, focou-se no trabalho de conscientização da paciente em relação aos seus hábitos de vida e higiene do sono; estes deveriam ser ajustados para a resolução da insônia. A paciente foi orientada a realizar as seguintes mudanças em seus hábitos de vida:

- Mudança do horário de atividade física de $21 \mathrm{~h}$ para o mais cedo possível. 
- Exclusão da ingestão de energéticos, cafeína e refrigerantes, idealmente 6 horas e, no mínimo, 4 horas antes do horário de ir para a cama.

- Não tirar cochilos maiores do que 45 minutos, exceto em situações de privação de sono na noite anterior.

- Mudança do horário de jantar de 22 para 20 horas e, no caso de fome em horários próximos a ir para cama, não dormir com fome, mas optar por uma refeição leve. Esta deveria ser, preferencialmente, um copo de leite ou uma banana, pois além de serem leves ajudam no sono.

- Mudança dos horários de estudo para antes do jantar e, no máximo, até 1 hora anterior a ir para a cama.

- Trocar o uso de televisão, computador ou celular por exercícios de relaxamento e respiração próximos ao adormecer.

- Horário de dormir adiantado de 23 para às 22 horas.

- Não ir para a cama sem sono e evitar a pressão sobre si para dormir. Na ausência de sono, além dos exercícios de relaxamento e respiração, é possível a prática de leitura, desde que não relacionada a trabalho ou concursos. A leitura não deve ser feita na cama. A cama deve ter seu uso restrito ao sono e à atividade sexual.

- Em situações de ausência de sono 20 minutos depois de ir para cama, levantar e voltar à prática de exercícios de relaxamento ou leitura. $\mathrm{O}$ mesmo procedimento deveria ser adotado se o sono se interrompesse durante a noite e não voltasse em até 20 minutos.

Embora a paciente apresentasse vários aspectos de sua vida que demonstrassem um jeito ansioso de pensar, o foco das primeiras seis sessões foi na compreensão do modelo cognitivo da insônia. Os dez minutos iniciais eram utilizados para a avaliação dos resultados da semana anterior; e os dez minutos finais de cada sessão para o treinamento da paciente em relação às técnicas de relaxamento e respiração.

As quatro sessões seguintes foram focadas no modelo cognitivo da ansiedade; e as duas últimas foram utilizadas para a avaliação do processo e certificação de que a paciente aprendeu a utilizar todos os aspectos terapêuticos, de forma autônoma. Uma $13^{\mathrm{a}}$ sessão foi realizada 30 dias após a $12^{\mathrm{a}}$, com o intuito de avaliar a efetividade do tratamento. Neste último encontro reaplicaram-se as Escalas de Beck, Epworth e IGI. Os resultados podem ser observados na (Figura 1). Contando com a primeira consulta, as 12 sessões de terapia e a última consulta foram no total de 14 encontros, que ocorreram no intervalo de quatro meses.

A partir da segunda sessão de terapia a paciente já relatou melhora na qualidade do sono; a contar da terceira sessão a paciente referiu melhora significativa do sono; e da quarta em diante a paciente relatou redução dos sintomas ansiosos. Os detalhes da evolução do tratamento estão apresentados na (Quadro 1). 
Research, Society and Development, v. 10, n. 16, e156101623441, 2021

(CC BY 4.0) | ISSN 2525-3409 | DOI: http://dx.doi.org/10.33448/rsd-v10i16.23441

Figura 1 - Avaliações dos sintomas de ansiedade e insônia pré e pós-intervenção.

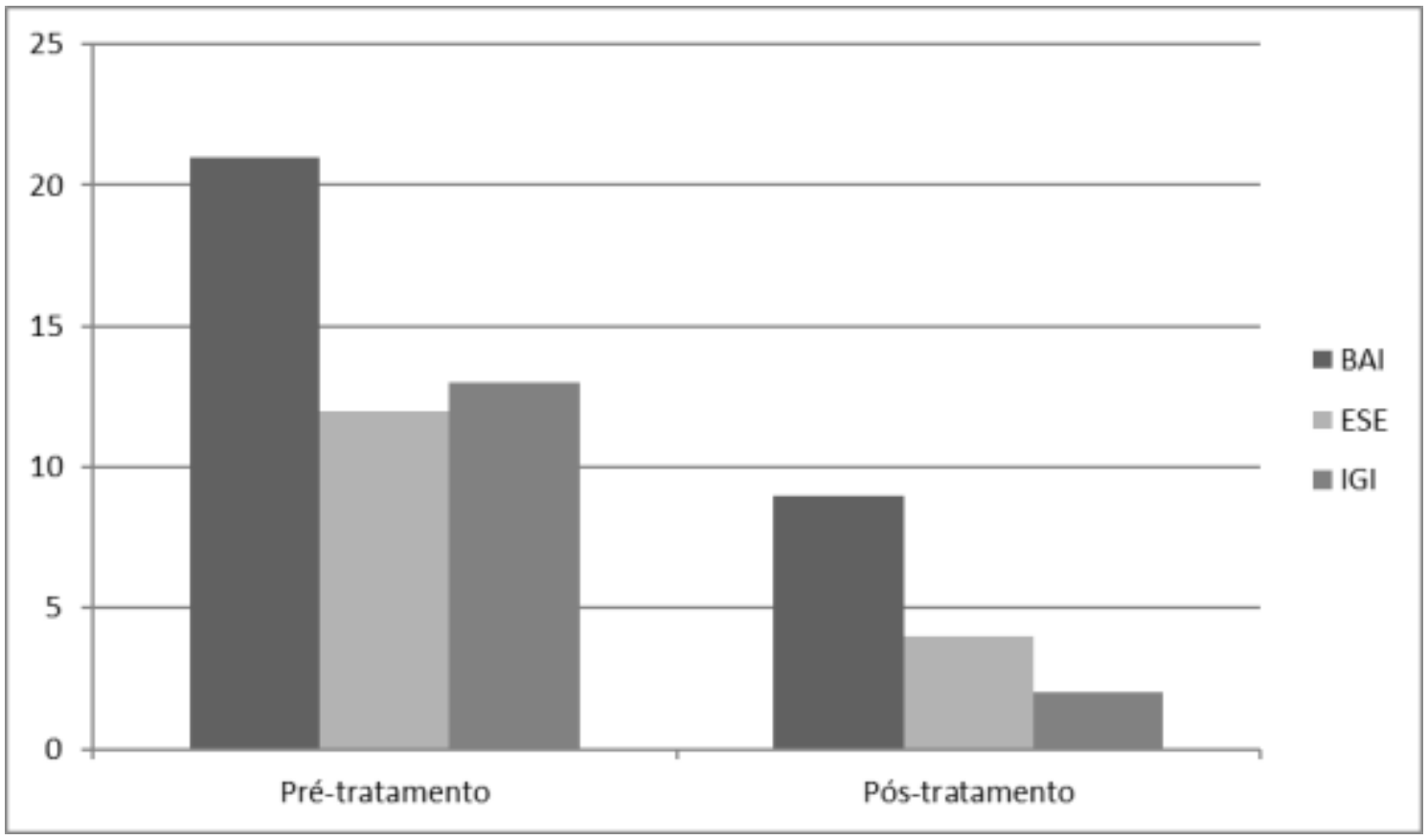

BAI = Beck Anxiety Inventory; ESE = Escala de Sonolência de Epworth; IGI = Índice de Gravidade de Insônia. Fonte: Autores (2021),

Quadro 1 - Evolução do tratamento.

\begin{tabular}{|c|c|c|}
\hline Sessão & Foco da sessão & Resultados observados \\
\hline Consulta inicial & $\begin{array}{l}\text { Queixa, anamnese, diário do Sono e } \\
\text { aplicação dos instrumentos de avaliação. }\end{array}$ & $\begin{array}{l}\text { Ansiedade moderada, sonolência diurna } \\
\text { excessiva e insônia. } \mathrm{BAI}=21 ; \mathrm{ESE}=12 ; \mathrm{IGI} \\
=13 \text {. }\end{array}$ \\
\hline $1^{\text {a }}$ Sessão & $\begin{array}{l}\text { Implementação da Higiene do Sono e } \\
\text { mudanças de hábitos de vida. }\end{array}$ & $\begin{array}{l}\text { Compromisso da paciente em relação às } \\
\text { mudanças de hábito e Higiene do Sono. }\end{array}$ \\
\hline $2^{\mathrm{a}}$ Sessão & $\begin{array}{l}\text { Início da TCC focada no modelo cognitivo } \\
\text { da insônia. }\end{array}$ & Relato de melhora no padrão de sono. \\
\hline $3^{\text {a }}$ Sessão & $\begin{array}{l}\text { Continuidade da TCC focada no modelo } \\
\text { cognitivo da insônia. }\end{array}$ & $\begin{array}{l}\text { Relato de melhora significativa do padrão de } \\
\text { sono. }\end{array}$ \\
\hline $4^{\text {a }}$ Sessão & $\begin{array}{l}\text { Continuidade da TCC focada no modelo } \\
\text { cognitivo da insônia. }\end{array}$ & Relato de redução dos sintomas ansiosos. \\
\hline $5^{\text {a }}$ Sessão & $\begin{array}{l}\text { Continuidade da TCC focada no modelo } \\
\text { cognitivo da insônia. }\end{array}$ & Normalização do padrão de sono \\
\hline $6^{\mathrm{a}}$ Sessão & $\begin{array}{l}\text { Fechamento da TCC focada no modelo } \\
\text { cognitivo da insônia. }\end{array}$ & $\begin{array}{l}\text { Consciência por parte da paciente em relação } \\
\text { ao modelo cognitivo da insônia. }\end{array}$ \\
\hline $7^{\text {a }}$ Sessão & $\begin{array}{l}\text { Início da TCC focada no modelo cognitivo } \\
\text { da ansiedade. }\end{array}$ & $\begin{array}{l}\text { Comprometimento da paciente para tarefas } \\
\text { visando redução da ansiedade. }\end{array}$ \\
\hline $8^{\text {a }}$ Sessão & $\begin{array}{l}\text { Continuidade da TCC focada no modelo } \\
\text { cognitivo da ansiedade. }\end{array}$ & $\begin{array}{l}\text { Sem mudanças significativas relatadas para } \\
\text { ansiedade. }\end{array}$ \\
\hline $9^{\text {a }}$ Sessão & $\begin{array}{l}\text { Continuidade da TCC focada no modelo } \\
\text { cognitivo da ansiedade. }\end{array}$ & $\begin{array}{l}\text { Relato de sensação de redução dos sintomas } \\
\text { de ansiedade. }\end{array}$ \\
\hline $10^{\mathrm{a}}$ Sessão & $\begin{array}{l}\text { Fechamento da TCC focada no modelo } \\
\text { cognitivo da insônia. }\end{array}$ & $\begin{array}{l}\text { Consciência por parte da paciente em relação } \\
\text { ao modelo cognitivo da ansiedade. }\end{array}$ \\
\hline $11^{\mathrm{a}}$ Sessão & $\begin{array}{l}\text { Avaliação dos resultados e fixação da } \\
\text { aprendizagem }\end{array}$ & $\begin{array}{l}\text { Relato de sensação de redução significativa } \\
\text { dos sintomas de ansiedade. }\end{array}$ \\
\hline $12^{\mathrm{a}}$ Sessão & $\begin{array}{l}\text { Avaliação dos resultados e fixação da } \\
\text { aprendizagem }\end{array}$ & $\begin{array}{l}\text { Consciência por parte da paciente em relação } \\
\text { ao modelo cognitivo da ansiedade e da } \\
\text { insônia, compromisso em manter os ganhos } \\
\text { obtidos com o processo. }\end{array}$ \\
\hline $13^{\mathrm{a}}$ Sessão & $\begin{array}{l}\text { Avaliação da efetividade do tratamento e } \\
\text { reaplicação dos instrumentos de avaliação. }\end{array}$ & $\begin{array}{l}\text { Ansiedade mínima. } \mathrm{BAI}=9 ; \mathrm{ESE}=4 ; \mathrm{IGI}= \\
2 .\end{array}$ \\
\hline
\end{tabular}

Fonte: Autores (2021). 


\section{Discussão}

Este relato de caso mostra a importância da anamnese e da avaliação adequadas para o diagnóstico e para a escolha da opção terapêutica mais adequada à paciente. No relato a mesma já havia sido tratada de ansiedade e estava iniciando um novo procedimento; este foi identificado como transtorno ansioso, não sendo considerada a possibilidade de transtorno de insônia decorrente de maus hábitos de vida e má Higiene do Sono. Contudo, é preciso reforçar que nem sempre é simples definir qual é o problema de base em pacientes com transtornos de ansiedade e de sono, pois alterações do sono ocorrem em ambos os quadros nosológicos (American Psychiatry Association, 2013).

Ao tratar desta relação do transtorno de insônia com outras doenças e transtornos mentais, o DSM 5 refere que "nem sempre a direção da relação é clara, podendo alterar-se ao longo do tempo; por essa razão, insônia comórbida é a terminologia preferida na presença de insônia coexistente com outra condição médica (ou transtorno mental). Com frequência, indivíduos com transtorno de insônia têm algum transtorno mental comórbido, particularmente transtornos de ansiedade, transtornos bipolares e depressivos. A insônia persistente representa um fator de risco ou um sintoma precoce de subsequente transtornos de ansiedade, bipolar, depressivo e relacionados ao uso de substâncias." (Harvey 2002).

No caso relatado, ambos os profissionais prenderam a sua atenção nos sintomas de ansiedade e não ativeram aos hábitos do sono da paciente, não chegando a considerar a possibilidade de um transtorno do sono. Outro ponto importante diz respeito ao rápido efeito que a Higiene do Sono e as mudanças de hábitos de vida provocaram na qualidade do sono da paciente. Efeito este que, potencializado pela TCC, fez com que ocorresse uma melhora significativa apenas duas semanas após o início da terapia, sendo que com três semanas de tratamento os sintomas ansiosos começaram a remitir, antes mesmo que o foco da terapia fosse transferido dos problemas de insônia para o de ansiedade. É comprovada a importância da Higiene do Sono no tratamento não farmacológico da insônia 12,13; pesquisadores e clínicos apontam a Higiene do Sono e a terapia cognitiva como as condutas não farmacológicas, estrategicamente, mais apropriadas no tratamento da insônia (Gomes et. al., 2011).

Outro ponto preponderante de reflexão diz respeito à decisão sobre o foco inicial do tratamento; no caso relatado, a decisão em se iniciar o tratamento focado na insônia deu-se pelo fato da paciente ter resistência ao uso de medicação e, principalmente, por razão da paciente já ter feito um processo de TCC para ansiedade previamente. Este último resultou na remissão dos sintomas de ansiedade, mas sem efeito significativo sobre a qualidade do sono. Tal decisão se mostrou correta quando, já nas primeiras semanas de tratamento, a paciente apresentou melhora na qualidade do sono e os sintomas de ansiedade diminuíram antes mesmo do foco da terapia ser direcionado ao tratamento da ansiedade. Este fato corrobora a literatura que aponta para o potencial eficaz do modelo cognitivo e da Higiene do Sono no tratamento da insônia (Ford et. al., 2020)

Destaca-se como um dos fatores de bom prognóstico a pronta adesão ao tratamento, por parte da paciente, proporcionando rápidos resultados terapêuticos. Apesar de esta adesão ser importante em todos os tipos de tratamento, ela se torna mais relevante ainda quando o indivíduo se torna ativo em seu processo terapêutico, como é o caso da TCC. Peixoto et al. defendem que a eficácia de qualquer tratamento depende estreitamente da adesão do paciente (Leite \& Vasconcellos 2003). Tal afirmação corrobora os trabalhos de outros autores que tratam da importância da adesão no tratamento da esquizofrenia (Leyfer, Ruberg \& Woodruff-Borden., 2006).

\section{Conclusão}

Diante do exposto, foi possível observar a importância do cuidado com a Higiene do Sono em pacientes com transtornos mentais que apresentam queixas de alterações no fenômeno de dormir. Uma vez que a alteração do sono é um sintoma muito comum em transtornos ansiosos, os clínicos não devem negligenciar a importância dos cuidados com os hábitos 
de vida do paciente que afetem a qualidade do seu sono. Como também, a sua avaliação deverá ser a mais integral e dinâmica possível, visando um tratamento multidisciplinar e com tomada de decisões que visem, em última análise, uma melhor qualidade de vida de seu paciente.

\section{Referências}

American Psychiatry Association. Diagnostic and Statistical Manual of Mental disorders - DSM-5. (5th.ed.), American Psychiatric Association, 2013, $189,225,263,363$ e 368.

Amorim, J. R., Silva, I. A., \& Shimizu, I. S. (2017) Avaliação da qualidade de sono em pacientes com câncer de mama em quimioterapia. Rev Bras Mast. $27(1): 3-7$.

Barros, M. B. A., et al. Relato de tristeza/depressão, nervosismo/ansiedade e problemas de sono na população adulta brasileira durante a pandemia de COVID19. Epidemol Serv Saúde

Beck, J. Terapia Cognitivo-comportamental: Teoria e Prática. (2a ed.), Artimed, 2016, 22, 23.

Borges, N. B., \& Angelotti, G. S. (2002), Ansiedade e Depressão em uma amostra de pacientes classificados como portando fatores psicológicos que afetam as condições médicas. Rev Estudos de Psicol. 19 (3) 15-22.

Feng F, Yu S, Wang Z, Wang J, Park J, Wilson G, Deng M, Hu Y, Yan B, \& Kong J. (2019) As intervenções não farmacológicas e farmacológicas aliviam os sintomas de insônia modulando uma rede compartilhada: Um estudo longitudinal controlado. Neuroimage Clin. 22: 101745. 10.1016 / j.nicl.2019.101745.

Ford, M. E., et al (2020) Non-pharmacological treatment for insomnia following acquired brain injury: A systematic review. Sleep Med Rev. 50:101255. 10.1016/j.smrv.2019.101255.

Gil, A. C. (2002) Como elaborar projetas de pesquisaiAntonio Carlos Gil. (4a ed.), Atlas.

Gomes, J. B., et al. (2011) Terapia cognitivo-comportamental com intervenção familiar para crianças e adolescentes com transtorno obsessivo-compulsivo: uma revisão sistemática. Rev. psiquiatr. Rio Gd. Sul. 33(2): 121-127.

Harvey AG. (2002) A cognitive model of insomnia. Behaviour Research and Therapy. (40) 869-893.

Hochman, B., Nahas, F. X., Filho, R. S. de O., \& Ferreira, L. M. (2005). Desenhos de pesquisa. Acta Cir. Bras. 20(2), 2-9. https://doi.org/10.1590/S010286502005000800002

Leite, S. N., \& Vasconcellos, M. P. C. (2003) Adesão à terapêutica medicamentosa: elementos para a discussão de conceitos e pressupostos adotados na literatura. Ciênc Saúde Coletiva. 8(3):775-82.

Leyfer, O. T., Ruberg, J. L., \& Woodruff-Borden, J. (2006) Examination of the utility of the Beck Anxiety Inventory and its factors as a screener for anxiety disorders. J Anxiety Disord. 20(4): 444-58.

Llorca, P. M., et al. (2005) Cost- -effectiveness analysis of schizophrenic patient care settings: impact of an atypical antipsychotic under long-acting injection formulation. Encephale. 31(2):235-46.

Manfro, G. G. (2005) Terapia cognitivo-comportamental no transtorno de pânico. Rev. Bras. Psiquiatr. 30 ( Suppl 2 ): s81-s87.

Margis, R. (2015) Terapia Cognitivo-Comportamental na Insônia. Rev Debates em Psiquiatr. 5, 22-27.

Matta, A., et al. (2010) Intervenções Cognitivo-Comportamentais no Transtorno de Humor Bipolar. Interamerican Journal of Psuchology. 44.

Muller, M. R., \& Guimarães S. S. I. (2007) mpacto dos transtornos do sono sobre o funcionamento diário e qualidade de vida. Estudos de Psicologia 24(4), 519-528, <http://www.scielo.br/scielo.php?script=sci_arttext\&pid=S0103-166X2007000400011\&lng=pt_BR\&nrm=iso>. http://dx.doi.org/10.1590/S0103$166 \times 2007000400011$.

Pafume, C. M., Oliveira, P. O., Carliana, M. M., \& Aparecida, C. C. (2014) Prejuízos nutricionais e distúrbios no padrão de sono de trabalhadores da Enfermagem. Rev. bras. enferm. 67(5): 832-842. http://www.scielo.br/scielo.php?script=sci_arttext\&pid=S0034-71672014000500832\&lng=en. https://doi.org/10.1590/0034-7167.2014670523.

Peixoto, C., et al. (2010) Impacto do perfil clínico e sociodemográfico na adesão ao tratamento de pacientes de um Centro de Atenção Psicossocial a Usuários de Álcool e Drogas (CAPSad). J. bras. psiquiatr. 59(4): 317-321.

Prado, C. E. P. (2016) Estresse ocupacional: causas e consequências. Rev Bras Med Trab. 14(3):285-9.

Rabenda, V., Reginster, J. Y. (2010) Positive impact of compliance to strontium ranelate on the risk of nonvertebral osteoporotic fractures. Osteoporos Int. 16. Souza, F. V. P., Toledo, M. A., \&Sousa, M. N. A. (2021) Aspectos do tratamento da insônia na Atenção Básica. Brazilian Applied Science Review. 5(1): 358371.

Thorpy, M. J. (2012) Classification of Sleep Disorders. Neurotherapeutics. 9(4):687-701. 